\title{
Las elecciones legislativas de 2004. Un análisis de las encuestas y de la gestión comunicativa en la campaña electoral: su proyección en la decisión de voto
}

\section{Salomé Berrocal}

Profesora de Teoría de la Comunicación y Teoría de la Información,

Universidad CEU San Pablo de Madrid

\section{Clara Fernández}

Investigadora del Departamento de Periodismo.

Universidad CEU San Pablo de Madrid

\section{Resumen:}

El 14 de marzo de 2004 se celebraron elecciones generales en España. Los resultados de estos comicios nos obligan a hablar de vuelco electoral, se pasó de la mayoría absoluta del Partido Popular a la victoria del Partido Socialista. Dichas elecciones estuvieron marcadas por distintos acontecimientos como el apoyo de España en la Guerra de Irak, el encuentro de Carod Rovira con miembros de ETA, y, en especial, el atentado terrorista islámico que tuvo lugar en Madrid el 11 de marzo, tres días antes de las elecciones. Las encuestas, que se realizaron en los años 2003 y 2004, sirven para conocer cómo influyeron estos hechos en la decisión de voto de los españoles. El trabajo, que se presenta en este artículo, analiza los principales sondeos de opinión, realizados antes y después de las elecciones, así como el comportamiento de las cadenas de televisión generalistas desde el 11 al 14-M, con el objeto de colaborar en encontrar una explicación a los resultados electorales y a la fluctuación electoral.

Palabras clave:

Política, partidos políticos, elecciones, España, campaña electoral, encuesta, sondeo, opinión, prensa, periodismo. 


\section{Abstract:}

On March the $14^{\text {th }} 2004$ general elections were held in Spain. Their results force us to talk about an electoral upset, the Popular Party's absolute majority turned into a victory for the Socialist Party. These elections were influenced by several events such us the Spanish support of the Iraq War, Carod Rovira's meeting with members of the terrorist group ETA, and especially, the Islamic terrorist attack which took place in Madrid on March the $11^{\text {th }}$, three days before the elections. The surveys that were done during 2003 and 2004 show how these facts influenced the Spaniards' decision to vote. For this reason, these opinion polls have helped to explain in this dissertation the origin of the votes that leaded José Luis Rodríguez Zapatero to the Presidence of the Government.

Key words:

Politics, political parties, elections, Spain, electoral campaign, survey, poll, opinion, press, journalism.

\section{Introducción}

Referirse a las elecciones conocidas como 14-M, supone mencionar unos comicios muy especiales para los españoles, puesto que, tres días antes de su celebración, tuvo lugar en Madrid el mayor atentado terrorista sufrido en España. Una acción criminal en la que murieron 199 personas y 1.400 resultaron heridas. La gestión de este atentado por parte del Gobierno fue lo que los medios de comunicación atribuyeron como causa de la derrota del Partido Popular y del vuelco electoral que se produjo en España. Autores como Casimiro García-Abadillo (2004) y Rosa María Artal (2004) ofrecen en sus obras, referidas al brutal atentado del 11 de marzo, una detallada descripción de los hechos en los que, en un primer momento, el Gobierno adjudicaba la autoría de los atentados a la banda terrorista ETA, posición que mantuvo hasta que se descubrieron pruebas que demostraban que la organización terrorista $\mathrm{Al}$ Qaeda, era la principal sospechosa.

La investigación, que se presenta, se basa en el análisis comparativo de las encuestas electorales publicadas en los años 2003 y 2004, con el objeto de conocer qué hechos próximos a la fecha electoral influyeron en los españoles para que en España se pasara de una mayoría absoluta del Partido Popular a la victoria del Partido Socialista Obrero Español. Esta aportación puede resultar valiosa en el campo de estudio de las influencias en la decisión de voto, así como en las investigaciones sobre volatilidad electoral, más aún si se tiene en cuenta que aún son escasos los trabajos realizados sobre las elecciones legislativas de marzo de 2004.

Los gráficos, que se muestran a lo largo del estudio, son mayoritariamente de elaboración propia, y se han utilizado, como fuentes de datos, las distintas empresas que realizan sondeos en nuestro país. Asimismo, dado que la televisión se ha convertido en la principal mediadora en el ámbito de la comu- 
nicación política, este estudio recoge un análisis del comportamiento de la audiencia en España durante los días 11 al 14 de marzo de 2004.

\section{Las encuestas electorales: la opinión de los españoles antes del 14-M}

El Diccionario de la Lengua Española (RAE, 1992: 614) define la encuesta como el "conjunto de preguntas tipificadas dirigidas a una muestra representativa, para averiguar estados de opinión o diversas cuestiones de hecho." Esta definición hace referencia a las encuestas en general. Sin embargo, algunos autores han relacionado estos estudios de opinión con las campañas electorales.

Para algunos sociólogos, como Isidoro Alonso Hinojal (1999: 208), las encuestas son el método casi exclusivo para conocer la realidad, de hecho, "se han transformado en el instrumento preferido de la confrontación política, entiéndase del condicionamiento sucesivo para la caza del voto." Este profesor profundiza en la relación entre las encuestas y la política, ya que en su opinión, la encuesta electoral pretende "conocer la intención del voto electoral, anticipar el resultado de las elecciones con una seguridad que la Estadística precisa." Tienen tanta importancia para los partidos políticos, que parece como si "en España estuviéramos en campaña electoral permanentemente."

Robert Worcester (1995: 192) coincide con Hinojal al afirmar que "los sondeos son de gran utilidad para los partidos políticos", ya que están "ansiosos por controlar en beneficio propio la información obtenida por los sondeos.” Por su parte, Ismael Crespo (2002: 58), define las encuestas electorales como "un instrumento muy útil para analizar la influencia de las campañas en la decisión de voto de los electores, entendida esta en un sentido amplio que incluye tanto la decisión de acudir a votar como la opción elegida." Crespo amplía la definición en el sentido en que no sólo les interesan a los partidos políticos los resultados de las encuestas para conocer la intención de voto de los ciudadanos, sino también para estudiar en ellos la influencia de las campañas electorales, así como para realizar una aproximación de la participación electoral de los votantes.

Siguiendo esta aportación de Crespo, se ha decidido estudiar, en este apartado, los resultados de los principales sondeos realizados antes y después de los comicios, teniendo en cuentas dos regulaciones significativas:

- la primera es la Ley Orgánica 5/1985, Régimen Electoral General, que se encarga de regular la difusión de estas encuestas, ya que estima que, "durante los cinco días anteriores al de la votación queda prohibida la publicación y difusión de sondeos electorales por cualquier medio de comunicación";

- la segunda, tiene que ver con la duración de la campaña electoral. La Ley Orgánica 5/1985, de 19 de junio de 1985, del Régimen Electoral General (LOREG), indica que la campaña electoral debe 
comenzar el día trigésimo octavo posterior a la convocatoria, durará quince días y terminará a las cero horas del día inmediatamente anterior a la votación.

\section{a) La utilidad de la campaña electoral}

El objeto de la investigación, en este epígrafe, es conocer a través de las encuestas cómo vivieron los españoles la proximidad de unas elecciones generales y qué importancia conceden a la campaña electoral. Hay que mencionar que la utilidad de las campañas electorales es debatida entre diferentes autores. "Para algunos, las elecciones se ganan o se pierden antes del día de la votación". Las campañas sirven en este caso, para "consolidar o amplificar las tendencias de voto". Para otros, "su utilidad radica en que moviliza al electorado fiel y pueden ser usadas para incidir entre los grupos de votantes indecisos" (Martínez; Méndez, 2004: 24).

Las campañas como función principal tienen la legitimación del sistema político (Crespo; Moreno, 2003: 24), pero los sondeos de opinión indican que las campañas son importantes, también, porque es la manera que tienen los partidos políticos de informar a los votantes sobre sus promesas electorales, sirven para consolidar la imagen pública del candidato de cada partido, o para darle a conocer en caso de que éste se presente a las elecciones por primera vez, como así ocurrió en las pasadas elecciones al Congreso de los Diputados del 14 de marzo de 2004, tanto en el caso de Mariano Rajoy por el Partido Popular (PP), como en el caso de José Luís Rodríguez Zapatero por parte del Partido Socialista Obrero Español (PSOE).

Mediante la encuesta postelectoral del CIS de 2004, podemos conocer cuál es la utilidad que tuvo la campaña electoral para los españoles.

\begin{tabular}{|l|c|c|c|c|}
\hline & $\begin{array}{c}\text { Informarse sobre } \\
\text { los programas y } \\
\text { propuestas de cada } \\
\text { partido o coalición }\end{array}$ & $\begin{array}{c}\text { Conocer mejor } \\
\text { a los candidatos }\end{array}$ & $\begin{array}{c}\text { Ver las diferencias } \\
\text { que existen entre } \\
\text { unos partidos } \\
\text { y otros }\end{array}$ & $\begin{array}{c}\text { Decidir } \\
\text { su voto }\end{array}$ \\
\hline Mucho & 3.3 & 2.8 & 3.0 & 2.1 \\
\hline Bastante & 27.8 & 29.2 & 29.5 & 14.2 \\
\hline Poco & $\mathbf{3 8 . 0}$ & $\mathbf{3 5 . 3}$ & $\mathbf{3 4 . 2}$ & 26.2 \\
\hline Nada & 26.2 & 28.0 & 28.4 & $\mathbf{5 2 . 4}$ \\
\hline N.S. & 4.6 & 4.5 & 4.7 & 4.4 \\
\hline N.C. & 0.1 & 0.2 & 0.3 & 0.6 \\
\hline
\end{tabular}

Fuente: CIS, Estudio n. ${ }^{\circ}$ 2.559, Postelectoral elecciones generales y autonómicas de Andalucía 2004. 
En este gráfico se observa que mientras el $31.1 \%$ de los encuestados afirma que la campaña electoral les ha servido mucho o bastante, para informarse sobre los programas y propuestas de cada partido o coalición, el $64.2 \%$ asegura que les ha valido de poco o nada. Otra de las funciones de las campañas electorales es dar a conocer mejor a los candidatos. En este sentido, frente al $32 \%$ que admite que les ha influido mucho o bastante, el $63.3 \%$ afirma que la campaña electoral les ha servido de poco o nada para este fin. Respecto a su utilidad para conocer las diferencias que existen entre unos partidos y otros, el $32.5 \%$ de los entrevistados confiesan que les sirve de mucho o bastante, mientras que al $63.3 \%$ poco o nada. Finalmente el $16.3 \%$ de los españoles creen que las campañas electorales les influye mucho o bastante a la hora de decidir su voto. No obstante, el 78,6 admite que les ha servido de poco o nada para optar por un partido o por otro.

Durante las campañas, "la información electoral trata de relatar y enseñar al público la actividad realizada por el partido en campaña en ese día” (Berrocal, 2003: 138). En esta cuestión, sobre el interés que demostraron los españoles en el seguimiento de la campaña electoral de las elecciones de 2004, el CIS proporciona dos sondeos realizados antes y después de los comicios.

\begin{tabular}{|l|c|c|c|c|c|c|}
\hline & $\begin{array}{c}\text { Con } \\
\text { mucho } \\
\text { interés }\end{array}$ & $\begin{array}{c}\text { Con } \\
\text { bastante } \\
\text { interés }\end{array}$ & $\begin{array}{c}\text { Ni con mucho, } \\
\text { ni con poco } \\
\text { interés }\end{array}$ & $\begin{array}{c}\text { Con } \\
\text { poco } \\
\text { interés }\end{array}$ & $\begin{array}{c}\text { Sin } \\
\text { ningún } \\
\text { interés }\end{array}$ & N.C. \\
\hline Preelectoral & $8.9 \%$ & $34.6 \%$ & - & $\mathbf{3 9 . 4 \%}$ & $16.7 \%$ & $0.3 \%$ \\
\hline Postelectoral & $12.1 \%$ & $\mathbf{3 3 . 4 \%}$ & $13.7 \%$ & $22.8 \%$ & $18.0 \%$ & $0.1 \%$ \\
\hline
\end{tabular}

Fuente: CIS.

Tabla: Elaboración propia.

Mientas que en la encuesta preelectoral (realizada días antes del atentado) el $43.5 \%$ afirmaba haber seguido la campaña con mucho o bastante interés y un $56,1 \%$ declaraban haberla seguido con poco o ningún interés, en la encuesta postelectoral (ejecutada después de la celebración de los comicios) el porcentaje de los encuestados interesados en la campaña electoral subió al $45.5 \%$, mientras que el de los poco o nada interesados bajó al $40.8 \%$. Resulta probable que influyera en este incremento la amplia cobertura, realizada entre los días 11 al $14 \mathrm{M}$, por la televisión, considerada principal mediadora en la comunicación política, y las declaraciones que sobre la autoría del atentado cruzaron los distintos líderes políticos.

A continuación, se puede observar el seguimiento realizado por las principales cadenas de televisión generalistas en nuestro país desde el 11 al 14 de marzo de 2004.

\section{b) Cambios en la programación y audiencia televisiva del 11 al 14-M}

El 11 de marzo todas las cadenas modificaron su parrilla para informar a los espectadores sobre el atentado en Madrid. Durante todo el día mantuvieron su programación habitual en la que incluyeron 


\begin{tabular}{|c|c|c|c|c|c|c|}
\hline Cadena & $\begin{array}{c}\text { Duración } \\
\text { minutos }\end{array}$ & Título / Descripción & Cuota & Miles & $\begin{array}{c}\text { Tiempo de } \\
\text { información } \\
\text { desde las } \\
08: 00 h .\end{array}$ & $\begin{array}{c}\text { Tiempo de } \\
\text { publicidad } \\
\text { desde las } \\
08: 00 h .\end{array}$ \\
\hline \multirow{8}{*}{ 牙 } & 76 & Informativos T5 matinal & 18,5 & 312 & \multirow{8}{*}{973} & \multirow{8}{*}{430} \\
\hline & 109 & La mirada crítica & 12,4 & 516 & & \\
\hline & 195 & Día a día & 18,3 & 1.227 & & \\
\hline & 65 & Informativos T5 14:30 & 18,8 & 2.669 & & \\
\hline & 260 & A tu lado / 11 de marzo de 2004, masacre en Madrid & 15,5 & 1.616 & & \\
\hline & 120 & Informativos T5 20:30 & 17,2 & 2.598 & & \\
\hline & 96 & Crónicas marcianas / 11M Masacre en Madrid & 40,1 & 3.074 & & \\
\hline & 52 & Informativos T5 noche & 48,5 & 1.254 & & \\
\hline \multirow{9}{*}{ 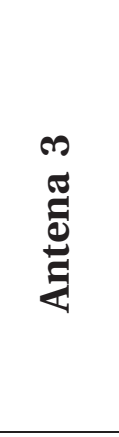 } & 347 & Noticias de la mañana & 20,3 & 712 & \multirow{9}{*}{1.054} & \multirow{9}{*}{307} \\
\hline & 59 & Antena 3 noticias avance / Matanza en Madrid & 19,2 & 2.375 & & \\
\hline & 52 & Antena 3 noticias 1 & 23,4 & 3.386 & & \\
\hline & 214 & Especial Sabor a ti / 11M Masacre en Madrid & 19,5 & 1.969 & & \\
\hline & 57 & Especial informativo / Masacre de ETA en Madrid & 17,7 & 1.946 & & \\
\hline & 75 & Antena 3 noticias 2 & 21,7 & 3.406 & & \\
\hline & 118 & Especial informativo / 11M Masacre en Madrid & 15,3 & 2.770 & & \\
\hline & 102 & Especial 7 días 7 noches / 11M Masacre en Madrid & 17,7 & 1.224 & & \\
\hline & 29 & Antena 3 noticias 3 & 15,7 & 326 & & \\
\hline \multirow{7}{*}{ PI } & 91 & Telediario matinal & 36,7 & 922 & \multirow{7}{*}{1.110} & \multirow{7}{*}{$\mathbf{0}$} \\
\hline & 12 & Los desayunos de TVE / José Luis Rodríguez Zapatero & 32,7 & 1.258 & & \\
\hline & 316 & Avance informativo / Matanza en Madrid & 35,9 & 2.413 & & \\
\hline & 90 & Telediario 1 & 35,1 & 4.857 & & \\
\hline & 267 & Especial informativo / 11M Matanza en Madrid & 29,4 & 3.041 & & \\
\hline & 104 & Telediario 2 & 22,2 & 3.977 & & \\
\hline & 88 & 11-M Matanza en Madrid & 16,9 & 2.831 & & \\
\hline
\end{tabular}

Fuente: SOFRES.

Tabla: Elaboración propia.

especiales informativos, tal y como se observa en la tabla, y alargaron la duración de sus informativos. En el caso de Antena 3, prolonga la duración de las Noticias de la mañana hasta las 13:47h., momento en el que incluye su primer bloque de publicidad. Este programa es el que tuvo mayor audiencia de la cadena, con un 20,3. Los programas Día a día (A3) y A tu lado (T5) incluyeron 3 y 4 avances informativos 
respectivamente. En horario de prime time ${ }^{1}$, Telecinco emitó la serie Los Serrano, mientras que el resto de las cadenas siguieron con sus informativos tratando el atentado. Lo más visto del día y de la cadena fue el Informativo T5 Noche, con un 48,5 de cuota de pantalla, seguido del programa Crónicas marcianas con un 40,1.

Por su parte TVE1, no emitió publicidad desde las 08:00hr, recortó la duración de Los desayunos de TVE, que tenía como invitado a José Luis Rodríguez Zapatero, para introducir un especial informativo desde las 09:43h. hasta el inicio del Telediario 1, que prolonga su duración hasta las 16:30h. aproximadamente. La tarde se dedicó íntegramente a un especial informativo hasta el comienzo del Telediario 2. Le sigue el último especial informativo hasta las 24:07h., cuando comienza el Canal 24H Noticias. El programa que trató los atentados del 11-M con mayor audiencia de TVE1 este día fue el avance informativo, con 35,9 de cuota, que comenzó a las 09:43h. y duró más de 3 horas. Mientras que TVE-1 fue la cadena que más minutos dedicó a la información (1.110 minutos desde las 08:00 horas), Telecinco fue la que más tiempo invirtió en publicidad (430 minutos) y menos en información (973 minutos).

El 12 de marzo, el día después del atentados, todas las cadenas mantuvieron la tónica informativa del día anterior hasta el prime time, donde se relaja la presión informativa para emitir cine. Los servicios informativos de las cadenas desplegaron todos sus recursos para cubrir las manifestaciones que tuvieron lugar ese día en distintos puntos de España. Telecinco centró su atención especialmente en Madrid, Barcelona y Euskadi, mientras que TVE1 y Antena 3 ampliaron sus coberturas a otros territorios. Antena 3 recuperó su política publicitaria, sin embargo, TVE1 suprimió todos sus anuncios hasta la franja de prime time.

El programa más seguido por los españoles este día fue el informativo Canal 24H Noticias, con una cuota del 34,8, que comenzó hacia la medianoche del 11 de marzo y terminó a las 07:30 de la mañana del día siguiente.

El 13 de marzo de 2004, en la jornada de reflexión, las tres cadenas nacionales volvieron a su programación habitual, siendo interrumpida por la comparecencia del ministro Ángel Acebes. A pesar de ser la misma señal para todas las cadenas, los telespectadores prefirieron seguir sus declaraciones en la cadena pública con una 32,4 de cuota, convirtiéndose así esta intervención en lo más visto del día.

El 14-M, fecha de convocatoria de las elecciones generales, presentó un espectacular incremento del consumo de información frente a las elecciones del año 2000.

${ }^{1}$ El prime time es una franja horaria que abarca desde las 21:00 hasta las 00:00 horas, en la que el consumo de televisión es mayor que el resto del día. 
Cuota de pantalla: datos total día

\begin{tabular}{|l|c|c|c|c|c|c|c|c|}
\hline & TTV $^{2}$ & TVE1 & Antena 3 & Telecinco & Autonómicas & La 2 & C + & Resto $^{\mathbf{3}}$ \\
\hline $\mathbf{1 2 / 0 3 / 2 0 0 0}$ & 5.904 & 24,1 & 20,3 & 18,2 & 18,1 & 9,1 & 3,1 & 7,1 \\
\hline $\mathbf{1 4 / 0 3 / 2 0 0 4}$ & 7.408 & $\mathbf{2 4 , 0}$ & 17,4 & 20,7 & 18,9 & 6,2 & 2,1 & 10,6 \\
\hline Diferencias & $\mathbf{1 . 5 0 4}$ & $-0,1$ & $-2,9$ & $\mathbf{2 , 5}$ & 0,8 & $-2,9$ & $-1,0$ & 3,5 \\
\hline
\end{tabular}

Fuente: SOFRES.

Tabla: Elaboración propia.

Tal y como se observa en el cuadro, el consumo de televisión en España el 14 de marzo de 2004 aumentó en 1.504.000 espectadores, en comparación con el 12 de marzo de 2000. De las cadenas nacionales, Televisión Española fue la cadena más seguida por los españoles con un 24,0 de cuota de pantalla, al igual que en las elecciones de 2000. Sin embargo, Telecinco fue la que más incrementó su consumo en relación al año 2000 con un aumento de 2,5 puntos de cuota de pantalla. En contraposición se encuentra Antena 3, cuya cuota descendió un 2,9 respecto a las elecciones del año 2000.

c) Dos acontecimientos que marcaron la agenda de la campaña electoral:

la guerra de Irak y las conversaciones de Carod Rovira con ETA

La participación de España en la guerra de Irak:

El País publicó el 7 de marzo de 2004, un sondeo del Instituto Opina en el que se preguntaba a los ciudadanos si influiría en su decisión de voto la decisión del Gobierno del PP de apoyar sin condiciones, junto a Bush y Blair, la intervención militar en Irak.

\begin{tabular}{|c|c|c|}
\hline Sí & No & N.S. / N.C. \\
\hline 37.5 & $\mathbf{5 2 . 9}$ & 9.6 \\
\hline
\end{tabular}

Fuente: Instituto Opina.

Tabla: Elaboración propia.

En el gráfico se observa que el 52.9\% de los españoles admiten que no les influirá en su decisión de voto, frente al $37.5 \%$ que indica que sí les influirá.

Por su parte, El Mundo difundió, el 5 de marzo de 2004, un estudio realizado por Sigma Dos en el que se ponderó la posibilidad de que la posición del Gobierno en la Guerra de Irak, era razón suficiente para no votar al PP.

2 TTV: Es la media de espectadores que vieron la televisión ese día.

${ }^{3}$ Resto: Se refiere a aquellas comunidades autonómicas de España que no cuentan con un canal autonómico. 
Recuerdo de voto

\begin{tabular}{|l|c|c|c|c|}
\hline & PSOE & PP & IU & Otros \\
\hline Sí & $74.1 \%$ & $17.2 \%$ & $\mathbf{8 0 . 7 \%}$ & $48.5 \%$ \\
\hline No & $23.5 \%$ & $\mathbf{7 7 . 1 \%}$ & $18.0 \%$ & $44.5 \%$ \\
\hline N.S. / N.C. & $2.3 \%$ & $5.6 \%$ & $1.3 \%$ & $7.0 \%$ \\
\hline
\end{tabular}

Fuente: Sigma Dos. Publicado en El Mundo el 5 de marzo de 2004.

Se observa que el $\mathbf{8 0 . 7 \%}$ de los votantes de IU junto con el $74.1 \%$ de los socialistas, piensan que el apoyo de España en la Guerra de Irak sí es razón para no votar al PP, mientras que el $77.1 \%$ de los populares creen que no es razón suficiente.

Una encuesta del CIS de abril de 2003 revelaba que el 75.3\% de los encuestados pensaba que la participación de España en la Guerra de Irak no podría hacerles cambiar su decisión de voto en las elecciones municipales y autonómicas del mes de mayo, frente al 19.2\% que pensaban que sí que le podía influir.

\begin{tabular}{|c|c|c|c|}
\hline Sí & No & No sabe & No contesta \\
\hline 19.2 & $\mathbf{7 5 . 3}$ & 4.8 & 7 \\
\hline
\end{tabular}

Fuente: CIS, Estudio n. ${ }^{\circ} 2.508$, abril 2003.

Según las encuestas del CIS, la participación de España en la Guerra de Irak, no era un factor que pudiera cambiar, de forma mayoritaria, la decisión de voto de los españoles.

\section{El encuentro de Carod Rovira con miembros de ETA:}

El diario $A B C$ publicó, el 26 y 27 de enero de 2004, que el 4 de enero se había celebrado un encuentro cerca de Perpiñán entre Carod-Rovira, secretario general de ERC, siendo conseller en cap del gobierno tripartito de Cataluña presidido por Pasqual Maragall, con los dirigentes de ETA: Mikel Albizu, Antza, y José Antonio Urrutikoetxea, Josu Ternera. (Campmany, 2005: 230)

El Instituto Opina realizó una encuesta en marzo de 2004, para conocer la opinión de los ciudadanos respecto a la repercusión de la reunión de Carod Rovira con miembros de ETA. Este estudio se publicó el 7 de marzo de 2004 en El País.

\begin{tabular}{|l|c|c|c|c|c|}
\hline & Favorece & Indiferente & Perjudica & No sabe & No contesta \\
\hline PP & $\mathbf{5 1 . 2}$ & 10.1 & 18.2 & 19.0 & 1.6 \\
\hline PSOE & 4.7 & 8.5 & $\mathbf{6 6 . 7}$ & 18.5 & 1.7 \\
\hline
\end{tabular}

Fuente: Instituto Opina. Encuesta España. Estimación electoral Marzo 2004.

Tabla: Elaboración propia. 
Siguiendo los resultados del sondeo, el 51.2\% creía que el encuentro de Carod Rovira con ETA favorecería al PP en las elecciones generales, el $18.2 \%$ pensaba que le podría perjudicar, y el $10.1 \%$ consideraba que era indiferente este hecho en la decisión de voto. Por otra parte, el $66.7 \%$ indicaba que este hecho podría perjudicar al PSOE, mientras que el $4.7 \%$ creía que favorecería al partido socialista.

Para comprobar el posible efecto en la decisión de voto, del encuentro mantenido en enero por Carod Rovira con ETA, se muestran los datos de intención de voto, según las encuestas realizadas por Sigma Dos en el mes enero y febrero.

\begin{tabular}{|l|c|c|c|c|}
\hline \multicolumn{1}{|c|}{2004} & PP & PSOE & IU & CIU \\
\hline Enero & $\mathbf{4 4 . 6}$ & 33.9 & 6.2 & 3.8 \\
\hline Febrero & $\mathbf{4 4 . 3}$ & 34.8 & 5.6 & 3.6 \\
\hline
\end{tabular}

Fuente: Sigma Dos.

Tablas: Elaboración propia.

La encuesta de Sigma Dos muestra que, en febrero, el PSOE no solo no sufre un descenso respecto al mes enero, debido a la crisis de Cataluña, sino que aumenta la intención de voto socialista. La ventaja que el PP tenía en enero era de 10.7 puntos, mientras que en febrero se reducía hasta los 9.5 puntos de diferencia.

\section{Los resultados electorales en las elecciones legislativas del 14-M}

En las elecciones generales del 14 de marzo de 2004, según la Oficina del Censo Electoral, los votantes tenían en su mayoría, entre 25 y 44 años, ya que representaban el $28.73 \%$ del censo electoral, además era mayor el porcentaje de votantes femeninas, concretamente el $51.48 \%$ frente al $48.52 \%$ de hombres, casi un millón más. El 21.78\% del electorado tenía más de 65 años, y 1.957 .912 jóvenes tuvieron la opción de votar por primera vez. Andalucía fue la comunidad autónoma con más electores nuevos. Respecto al censo de las generales anteriores, el censo creció en 603.712 personas, y en 177.400, en relación a los comicios municipales del 25 de mayo de 2003, según datos del Instituto Nacional de Estadística. (Rodríguez, 2004: 25)

En la siguiente tabla se muestran los resultados electorales de los comicios de 2004, así como los del año 2000. 


\begin{tabular}{|l|c|c|c|c|c|c|}
\hline & \multicolumn{7}{|c|}{$\mathbf{2 0 0 4}$} & \multicolumn{3}{c|}{$\mathbf{2 0 0 0}$} \\
\hline PSOE & Escaños & Votos & $\mathbf{\%}$ & Escaños & Votos & \% \\
\hline PP & $\mathbf{1 6 4}$ & $\mathbf{1 0 . 9 0 9 . 6 8 7}$ & $\mathbf{4 2 . 6 4}$ & 125 & 7.918 .752 & 34.16 \\
\hline CIU & 148 & 9.630 .512 & 37.64 & $\mathbf{1 8 3}$ & $\mathbf{1 0 . 3 2 1 . 1 7 8}$ & $\mathbf{4 4 . 5 2}$ \\
\hline ERC & 10 & 829.046 & 3.24 & 15 & 970.421 & 4.19 \\
\hline EAJ-PNV & 8 & 649.999 & 2.54 & 1 & 194.715 & 0.84 \\
\hline IU & 7 & 417.154 & 1.63 & 7 & 353.953 & 1.53 \\
\hline CC & 5 & 1.269 .532 & 4.96 & 9 & 1.382 .333 & 5.96 \\
\hline BNG & 3 & 221.034 & 0.86 & 4 & 248.261 & 1.07 \\
\hline CHA & 1 & 205.613 & 0.8 & 3 & 306.268 & 1.32 \\
\hline EA & 1 & 93.865 & 0.37 & 1 & 75.356 & 0.33 \\
\hline Na-Bai & 1 & 60.613 & 0.32 & 1 & 100.742 & 0.43 \\
\hline
\end{tabular}

Fuente: El País, 16 de marzo de 2004, p. 35.

El PSOE, con respecto a las elecciones de 2000, ganó 2.990 .935 votos, es decir, un $37.7 \%$. Mientras que el PP perdió 690.666 sufragios en cuatro años. En el 2000, el Partido Popular tuvo una ventaja de 3.7 millones de votos, con la que obtuvo 183 escaños frente a los 125 que consiguió el PSOE. Esta diferencia cambia en 2004, ya que la superioridad de 1.3 millones de votos para el partido socialista, le adjudican 164 diputados, 39 más que en 2000, mientras que los populares han obtenido 148, después de haber perdido 35 diputados.

En cuanto a la participación electoral, las elecciones generales de 2004 se caracterizaron por su alto índice de participación. En la tabla, que se presenta a continuación, se puede observar la evolución de la participación y la abstención electoral en España.

\begin{tabular}{|l|c|c|c|c|c|c|c|}
\hline & $\begin{array}{c}\text { Junio } \\
\mathbf{1 9 7 7}\end{array}$ & $\begin{array}{c}\text { Marzo } \\
\mathbf{1 9 7 9}\end{array}$ & $\begin{array}{c}\text { Octubre } \\
\mathbf{1 9 8 2}\end{array}$ & $\begin{array}{c}\text { Junio } \\
\mathbf{1 9 8 6}\end{array}$ & $\begin{array}{c}\text { Marzo } \\
\mathbf{1 9 9 6}\end{array}$ & $\begin{array}{c}\text { Marzo } \\
\mathbf{2 0 0 0}\end{array}$ & $\begin{array}{c}\text { Marzo } \\
\mathbf{2 0 0 4}\end{array}$ \\
\hline Participación & $78.83 \%$ & $68.04 \%$ & $\mathbf{7 9 . 9 7 \%}$ & $70.49 \%$ & $77.38 \%$ & $68.71 \%$ & $77.23 \%$ \\
\hline Abstención & $21.17 \%$ & $\mathbf{3 1 . 9 6 \%}$ & $20.03 \%$ & $29.51 \%$ & $22.62 \%$ & $31.29 \%$ & $22.77 \%$ \\
\hline
\end{tabular}

Fuente: El País, 15 de marzo de 2004, p. 14.

Como se observa en el gráfico, las elecciones de 2004 no se caracterizan por tener el índice de participación más alto de su historia. Acudieron a votar el 77,23\% de españoles, mientras que en 1982, 
acudieron a las urnas el $79.97 \%$. Se esperaba una participación mayor ya que a las 14:00 horas, según el Ministerio del Interior, había votado el $41 \%$ del censo. Dado que la mitad del electorado, aproximadamente, acude a votar después de esa hora, "todas las previsiones apuntaban a que se superaría el 81\% de participación” (Garea, 2004: 8). No obstante, sí que fue notable la diferencia con respecto a las elecciones de 2000 , en las que el $68.71 \%$ de los ciudadanos ejercieron su derecho a voto.

\section{Las encuestas electorales: la opinión de los españoles después del 14-M}

\section{La fidelidad política}

Según una encuesta del CIS (Cacho, 1999: 20), el 9\% de los votantes que se consideran de derechas jamás estarían dispuestos a votar al PSOE, mientras que el $20 \%$ de los electores considerados de izquierdas asegura que nunca votaría al PP. Por ello, hay sociólogos que afirman que, en nuestro país, los votantes de los partidos de izquierda son más fieles que los votantes de derechas.

Después de las elecciones, el CIS quiso analizar las causas por las que los votantes optaron por el Partido Socialista o por el Partido Popular. En la siguiente tabla se deduce que los votantes del PSOE pueden ser considerados más fieles ya que lo eligen porque siempre le votan (30.1\%), o porque es el partido que mejor representa sus ideas (28.9\%). Sin embargo, los votantes populares se basan en razones más volátiles como por ejemplo, que sea el partido más capacitado para gobernar (23.1\%), o porque sus electores piensan que este partido lo ha hecho bien al frente del Gobierno (36.5\%).

\begin{tabular}{|c|c|c|c|}
\hline PP & $\mathbf{\%}$ & PSOE & $\mathbf{\%}$ \\
\hline Por Mariano Rajoy & 3.8 & Por J. L. Rodríguez Zapatero & 6.1 \\
\hline $\begin{array}{c}\text { Por los candidatos presentados } \\
\text { en mi provincia }\end{array}$ & 1.2 & $\begin{array}{c}\text { Por los candidatos presentados } \\
\text { en mi provincia }\end{array}$ & 0.7 \\
\hline $\begin{array}{c}\text { Por los atentados del 11-M en Madrid } \\
\text { y sus consecuencias }\end{array}$ & 0.8 & $\begin{array}{c}\text { Por los atentados del 11-M en Madrid } \\
\text { y sus consecuencias }\end{array}$ & 9.0 \\
\hline $\begin{array}{c}\text { Para evitar un gobierno de coalición entre } \\
\text { PSOE e IU }\end{array}$ & 2.3 & Para evitar que ganase el PP & 10.3 \\
\hline Porque es mi partido (siempre le voto) & 13.5 & Porque es mi partido (siempre le voto) & $\mathbf{3 0 . 1}$ \\
\hline $\begin{array}{c}\text { Porque es el que está más capacitado } \\
\text { para gobernar }\end{array}$ & $\mathbf{2 3 . 1}$ & $\begin{array}{c}\text { Porque es el que está más capacitado } \\
\text { para gobernar }\end{array}$ & 2.9 \\
\hline $\begin{array}{c}\text { Porque es el que mejor representa } \\
\text { las ideas de gente como yo }\end{array}$ & 15.4 & $\begin{array}{c}\text { Porque es el que mejor representa } \\
\text { las ideas de gente como yo }\end{array}$ & $\mathbf{2 8 . 9}$ \\
\hline $\begin{array}{c}\text { Porque lo ha hecho bien al frente } \\
\text { del Gobierno }\end{array}$ & $\mathbf{3 6 . 5}$ & $\begin{array}{c}\text { Por la posibilidad de que haya } \\
\text { un gobierno de izquierdas }\end{array}$ & 7.1 \\
\hline
\end{tabular}

Fuente: CIS.

Tabla: Elaboración propia. 
En las encuestas del CIS, posteriores a las elecciones de 2004, se puede analizar la fidelidad política de los españoles. Los encuestados, tras haberles preguntado a qué partido votaron el 14-M, respecto a los que mencionaron un partido en concreto (todos excepto "no tenía edad para votar", "en blanco", "no votó", "no recuerda" o "N.C.”), la mayoría confesaron que solían votar siempre por él, como se comprueba en esta tabla.

\begin{tabular}{|l|c|c|c|c|c|c|c|c|c|}
\hline \multicolumn{1}{|c|}{ Año 2004 } & Marzo & Abril & Mayo & Junio & Julio & Sept. & Oct. & Nov. & Dic. \\
\hline Era la primera vez que votaba & 16.0 & 12.2 & 12.2 & - & 10.9 & - & 11.6 & 11.6 & 12.9 \\
\hline Ya le había votado antes & 30.5 & 30.1 & 31.0 & - & 34.2 & - & 30.8 & 29.0 & 28.6 \\
\hline Suele votar siempre por él & $\mathbf{5 2 . 4}$ & $\mathbf{5 6 . 0}$ & $\mathbf{5 4 . 3}$ & - & $\mathbf{5 3 . 1}$ & - & $\mathbf{5 5 . 6}$ & $\mathbf{5 8 . 1}$ & $\mathbf{5 6 . 5}$ \\
\hline N.C. & 1.1 & 1.7 & 2.5 & - & 1.8 & - & 2.0 & 1.3 & 1.9 \\
\hline
\end{tabular}

Fuente: CIS.

Tabla: Elaboración propia.

En marzo, mes en el que se celebraron las elecciones, el $52.4 \%$ de los encuestados admitieron que el 14-M votaron al partido o coalición al que suelen elegir siempre. El mes con más fieles declarados fue noviembre con un $58.1 \%$.

La encuesta postelectoral de las elecciones legislativas de 2004 del CIS ofrece un sondeo con las opciones de los electores, en el caso de que hubieran sabido los resultados de estos comicios.

\begin{tabular}{|c|c|c|c|c|}
\hline $\begin{array}{c}\text { Hubiera votado } \\
\text { por el mismo } \\
\text { partido o coalición }\end{array}$ & $\begin{array}{c}\text { Se habría abstenido } \\
\text { o habría votado } \\
\text { en blanco }\end{array}$ & $\begin{array}{c}\text { Hubiera votado } \\
\text { por un partido } \\
\text { o coalición distinto }\end{array}$ & $\begin{array}{c}\text { Hubiera votado } \\
\text { por un partido en } \\
\text { vez de abstenerse }\end{array}$ & N.C. \\
\hline $\mathbf{8 6 . 2 \%}$ & $8.7 \%$ & $1.3 \%$ & $1.3 \%$ & $2.5 \%$ \\
\hline
\end{tabular}

Fuente: CIS, Estudio n. ${ }^{\circ}$ 2.559, Postelectoral elecciones generales y autonómicas de Andalucía 2004.

Según los datos presentados, el $86.2 \%$ de los electores habrían votado por el mismo partido o coalición, por lo tanto no se arrepienten de su decisión de voto, el $8.7 \%$ se habría abstenido o habría votado en blanco, mientras que el 1.3\% habría votado por un partido o coalición distinto, y otro $1.3 \%$ habría optado por un partido en vez de abstenerse.

\section{La fluctuación electoral}

La fluctuación electoral corresponde al número de electores que cambian su opción política de unas elecciones a otras. En el caso de las elecciones de marzo de 2004, y siguiendo el estudio postelectoral 
del CIS, a la pregunta ipodría decirme entre qué dos partidos u opciones dudó Usted? Las respuestas mayoritarias fueron las siguientes:

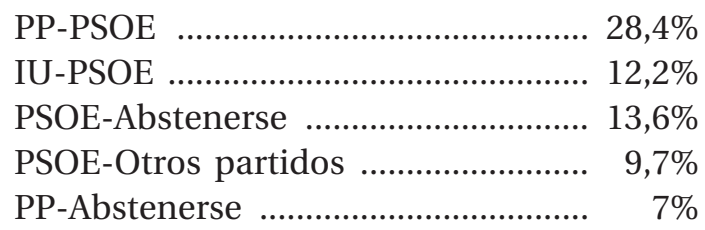

Un $28,4 \%$ de los entrevistados que dudaron, entre dos opciones, lo hicieron entre votar al Partido Popular o al Partido Socialista, un 12,2\% dudaron entre Izquierda Unida y el Partido Socialista, un 13,6\% entre el PSOE y la abstención, un 9,7\% entre el PSOE u otros partidos. Hay que destacar que en las cuatro respuestas mayoritarias, en las que el elector se plantea cierta dificultad en decidirse, se menciona como posibilidad el voto para el Partido Socialista.

\section{La influencia del atentado del 11-M en la decisión de voto}

Hasta la fecha, distintos sondeos se han ocupado de estudiar cuál fue el efecto del atentado terrorista en Madrid el 11 de marzo de 2004, tres días antes de la celebración de las elecciones legislativas en España. En este apartado se incluyen aquellas encuestas que se han ocupado de preguntar a los electores cómo les influyó el ataque terrorista en su decisión de voto.

Respecto a si el atentado del 11-M afectó mucho, bastante, poco o nada en la decisión de voto, esta es la valoración de los encuestados por el CIS.

\begin{tabular}{|c|c|c|c|}
\hline Mucho & Bastante & Poco & Nada \\
\hline $12.8 \%$ & $14.3 \%$ & $7.5 \%$ & $\mathbf{6 5 . 1 \%}$ \\
\hline
\end{tabular}

Fuente: CIS, Estudio n. ${ }^{\circ}$ 2.559, Postelectoral elecciones generales y autonómicas de Andalucía 2004.

Según la encuesta postelectoral del CIS, al $65.1 \%$ no les influyó nada los atentados del 11-M en su decisión de voto, junto con el 7.5\% que admitió que les influyó poco. Sin embargo, al 14.3\% de los encuestados el 11-M les influyó bastante y al 12.8\% mucho.

A los que admitieron que el 11-M influyó en su decisión de voto, se les preguntó en qué sentido.

\begin{tabular}{|c|c|c|}
\hline $\begin{array}{c}\text { Me animó a ir a votar, } \\
\text { ya que no pensaba hacerlo }\end{array}$ & $\begin{array}{c}\text { Cambié el voto del partido } \\
\text { al que pensaba votar }\end{array}$ & $\begin{array}{c}\text { Me reafirmó en la intención de } \\
\text { votar al que pensaba hacerlo }\end{array}$ \\
\hline $17.5 \%$ & $12.2 \%$ & $\mathbf{6 0 . 9 \%}$ \\
\hline
\end{tabular}

Fuente: CIS, Estudio n. ${ }^{\circ}$ 2.559, Postelectoral elecciones generales y autonómicas de Andalucía 2004. 
Al 60.9\% le reafirmó en la intención de votar al partido o coalición al que pensaba hacerlo, mientras que al $17.5 \%$ le animó a ir a votar, ya que no pensaba hacerlo. El $12.2 \%$ admitió que tras los atentados cambió el voto del partido al que pensaba votar.

Junto al CIS, Gallup realizó la misma encuesta respecto a si el 11-M influyó en la decisión de voto. En el siguiente cuadro se aprecian los datos obtenidos.

\begin{tabular}{|l|c|c|c|c|c|}
\hline & & \multicolumn{3}{|c|}{ PARTIDO VOTADO EN E.G. 2004 } \\
\hline & TOTAL & PSOE & PP & IU & OTROS \\
\hline No le influyó & 86.8 & $\mathbf{8 4 . 4}$ & $\mathbf{9 1 . 4}$ & $\mathbf{8 0 . 0}$ & $\mathbf{8 3 . 9}$ \\
\hline Le hizo cambiar lo que tenía pensado votar & 3.8 & 4.5 & 2.6 & 10.0 & 3.0 \\
\hline $\begin{array}{l}\text { Le ayudó a decidirse por una de las opciones de voto } \\
\text { entre las que dudaba }\end{array}$ & 8.3 & 10.5 & 5.1 & 10.0 & 9.5 \\
\hline N.S. I N.C. & 1.1 & 0.6 & 0.9 & - & 3.5 \\
\hline
\end{tabular}

Fuente: Gallup.

Tabla: Elaboración propia.

Como se observa en la tabla, al $84.4 \%$ de los votantes socialistas el atentado del 11-M no les influyó, junto con el $91.4 \%$ de los populares y el $80.0 \%$ de los electores de Izquierda Unida. Pero, el atentado tuvo repercusión en algunos españoles a la hora de cambiar su decisión de voto respecto al partido que tenían pensado votar, el $4.5 \%$ de los que votaron al PSOE no tenía pensado hacerlo, así como el $2.6 \%$ de los votantes del PP y el $10.0 \%$ de los electores de IU. Se deduce, por tanto, que el $14.5 \%$ de los españoles que votaron por al PSOE e IU no tenían pensado hacerlo antes del 11 de marzo de 2004. Como última opción el 11-M ayudó al $10.5 \%$ de los votantes socialistas a decidirse por una de las opciones de voto entre las que dudaba, así como el $5.1 \%$ de los populares y el $10.0 \%$ de los electores de IU. Estos datos apuntan a que el $20.5 \%$ de los españoles que votaron al PSOE e IU dudaban si votar a estos partidos o no antes del atentado.

Según la encuesta postelectoral del CIS, el atentado del 11 de marzo tuvo también otros efectos sobre los votantes, respecto a cuándo decidieron votar al partido o coalición electoral al que finalmente votó.

\begin{tabular}{|c|c|c|c|}
\hline $\begin{array}{c}\text { Lo tenía decidido hace tiempo } \\
\text { (antes de la campaña) }\end{array}$ & $\begin{array}{c}\text { Lo decidió durante la campaña, } \\
\text { antes del atentado del 11-M }\end{array}$ & $\begin{array}{c}\text { Lo decidió después } \\
\text { del atentado del 11-M }\end{array}$ & N.C. \\
\hline $\mathbf{8 7 . 1}$ & 3.4 & 9.2 & 0.3 \\
\hline
\end{tabular}

Fuente: CIS, Estudio n. ${ }^{\circ}$ 2.559, Postelectoral elecciones generales y autonómicas de Andalucía 2004. 
Se aprecia en la tabla que el $\mathbf{8 7 . 1 \%}$ de los votantes que fueron a votar lo tenía decidido antes de la campaña. El 3.4\% lo decidió durante la campaña, antes del atentado del 11-M, mientras que el $9.2 \%$ lo decidió después del 11-M.

Volviendo a las razones por las que los electores optaron por votar al PP o al PSOE, que ya se han mencionado en el apartado sobre la fidelidad política, se observan los siguientes datos en relación a la influencia de los atentados en Madrid en la decisión de voto.

\begin{tabular}{|c|c|c|c|}
\hline PP & $\mathbf{\%}$ & PSOE & $\mathbf{\%}$ \\
\hline $\begin{array}{c}\text { Por los atentados del 11-M en Madrid } \\
\text { y sus consecuencias }\end{array}$ & 0.8 & $\begin{array}{c}\text { Por los atentados del 11-M en Madrid } \\
\text { y sus consecuencias }\end{array}$ & $\mathbf{9 . 5}$ \\
\hline
\end{tabular}

Fuente: CIS.

Tabla: Elaboración propia.

Se aprecia que el 9.5\% de los votantes socialistas eligieron al partido que lidera Rodríguez Zapatero por los atentados del 11-M y sus consecuencias, mientras que al $0.8 \%$ de los populares les influyeron estas circunstancias.

No obstante, la incógnita no es tanto si influyó el atentado en Madrid el 11 de marzo en las elecciones del 14-M, sino la gestión del gobierno popular respecto a este asunto. Según el periodista Ernesto Ekaizer, tanto el atentado terrorista como, sobre todo, la conducta del Gobierno de José María Aznar ante él, sirvieron para refrescar la memoria al electorado de la guerra de Irak, pasando así una doble factura al Partido Popular en las urnas. Ekaizer afirma que "el Gobierno apostó por una referencia en la autoría de la masacre del 11-M: ETA", ya que la opcción islámica recordaría el pasado, la participación de España en la guerra de Irak. (Ekaizer, 2004: 22)

Por su parte, Belén Barreiro, opina que un atentado terrorista puede tener diversos efectos en la opinión pública de cara a unas elecciones. En el caso de España, se comentó la posibilidad de que los ciudadanos, "compungidos ante el horror, se volcaron a favor del cambio". Pero "el terror no produce deseo de cambio sino más bien todo lo contrario”(Barreiro, 2005: 16). Sin embargo, hay otra hipótesis respecto la autoría del crimen. Barreiro afirma que "éste fue el razonamiento del Gobierno y quizá también el de la oposición. Si ETA era responsable, el PP ganaba. Si el atentado había sido perpetrado por islamistas, el PP perdía” (Barreiro, 2005: 17).

Según datos ofrecidos por el Instituto Opina (Barreiro. 2005: 17), los electores no castigaron el atentado, sino la reacción del Gobierno ante los mismos. De este modo, el $60 \%$ de los españoles cree que "el Ejecutivo no informó correctamente sobre la autoría de los crímenes y no ofreció toda la información disponible".

Más de un año después de aquel fatídico día, todavía se sigue analizando cuál fue su repercusión en las elecciones. El Mundo publicó el 13 de marzo de 2005 un sondeo de Sigma Dos según el cual, el 
69\% de los españoles piensa que el PSOE no habría ganado las elecciones el 14 de marzo, de no haberse producido el atentado en Madrid tres días antes.

\section{Otros acontecimientos que pudieron marcar la decisión de voto:} El Plan Hidrológico Nacional y el Prestige

\section{El Plan Hidrológico Nacional}

En Aragón, en las elecciones de 2000, el Partido Popular obtuvo el 47.2\% de los votos, frente al 31.1\% del Partido Socialista. Con datos oficiales de los resultados de las elecciones de 2004, el PSOE aumentó un 42.5\% en Aragón respecto a las elecciones anteriores, mientras el Partido Popular sufrió un descenso del 17.1\%.

\section{El Prestige:}

En el año 2000, el Partido Popular fue la fuerza más votada en Galicia. Sin embargo, en las elecciones legislativas de 2004, los populares perdieron cuatro escaños que ganaron los socialistas. En el cómputo general, esto supone ocho escaños de diferencia a favor del PSOE.

Tras el escrutinio de los votos de las elecciones de 2004, se comprobó que el PSOE tuvo un aumento del $67.5 \%$ en Galicia respecto a 2000, mientras que el PP descendió un $8.5 \%$.

\section{Conclusiones}

1. En las elecciones generales del 14-M, los españoles encontraron poca utilidad en la llamada “campaña electoral”. Como en anteriores elecciones, sólo un tercio reconoció aprovechar este tiempo para informarse de los programas de los partidos o coaliciones, conocer mejor a los candidatos u observar las diferencias entre unos partidos y otros, y tan sólo un 16\% afirmó tener en cuenta el desarrollo de la campaña para decidir su voto.

2. La campaña fue seguida, según la encuesta postelectoral del CIS, con mucho o bastante interés por un $45 \%$ de los encuestados, frente a un $40 \%$ de entrevistados que declararon estar poco o nada interesados en el transcurso de la campaña.

3. El 11 de marzo de 2004 todas las cadenas modificaron su parrilla de programación, para informar a los espectadores sobre el atentado en Madrid. El 12 de marzo se mantuvo la tónica informativa. El 13 de marzo, en la jornada de reflexión, todas las cadenas nacionales volvieron a su programación habitual, siendo interrumpida por la comparecencia del ministro Ángel Acebes. El 14 de marzo, día de los comicios, el consumo televisivo aumento en 1.504 .000 espectadores, si comparamos con el consumo televisivo de las elecciones generales de 2000. 
La cadena generalista que más incrementó su consumo en el 14-M, respecto a las elecciones de 2000, fue Telecinco, con un aumento de 2,5 puntos de cuota de pantalla; aunque la cadena más vista, al igual que sucedió en el año 2000, fue Televisión Española.

4. Tanto la participación de España en la guerra de Irak, como el encuentro de Carod Rovira con la banda terrorista ETA, se consideraron acontecimientos de interés en la agenda de la campaña, pero no existe unanimidad en los sondeos sobre su posible influencia en la decisión de voto. Aunque, sí que en el caso de la intervención en Irak, cuando se aproxima el día de la votación, los sondeos comienzan a mostrar que un tercio de los encuestados tendrán en cuenta la guerra en su decisión de voto.

5. Según las encuestas analizadas, los votantes socialistas son más fieles a su partido político que los electores populares. En 2004 a la pregunta de porqué votaron a ese partido, los votantes del PSOE señalaron porque siempre le votan $(30.1 \%)$, o porque es el partido que mejor representa sus ideas (28.9\%). Los votantes del PP eligen a su partido por razones más volátiles, puesto que el 36.5\% eligieron esta opción porque pensaban que era el partido más capacitado para gobernar, junto con el $23.1 \%$ que votaron al Partido Popular porque pensaron que lo había hecho bien al frente del Gobierno.

6. En cuanto a la fluctuación electoral, parece que aquellos que dudaban entre dos partidos u opciones, respondieron de forma mayoritaria tener en cuenta al Partido Socialista. Esta situación nos conduce a pensar que fue la opción beneficiada en las dudas que presentaban los votantes.

7. Las investigaciones realizadas, sobre la posible influencia del atentado del 11-M en la decisión de voto, indican que a un $27 \%$ les afectó mucho o bastante, frente a un $65 \%$ a los no les influyó en su decisión de voto. De aquellos que respondieron haberse sentido influidos por el ataque terrorista, la mayoría (61\%) se reafirmó en su decisión de voto, mientras que un $17,5 \%$ se animó a votar o un 12,2\% cambió su voto. El 9.5\% de los votantes socialistas admitieron haber elegido a este partido por el atentado y sus consecuencias, mientras que el $0.8 \%$ de los votantes populares optaron por el PP por esta razón. De hecho, el 87.1\% de los entrevistados tenía decidido su voto antes del 11-M pero un 9,2\% lo decidió después del atentado.

8. Siguiendo los datos referidos a resultados electorales de 2004 y a las encuestas del CIS, éstas son algunas de las claves de la victoria del Partido Socialista:

- El PSOE resultó beneficiado por la volatilidad electoral. Resulta posible pensar que un pequeño porcentaje de los votantes del Partido Popular en 2000 cambiaran su decisión de voto en 2004, y lo dirigieran al Partido Socialista. Lo mismo cabría indicar de Izquierda Unida, que sufrió un retroceso en los comicios de 2004, y cuya opción más probable a la hora de cambiar de voto era el partido de Rodríguez Zapatero. 
- El aumento de participación electoral favoreció al PSOE. Mientras el Partido Popular perdió 690.666 electores en 2004, el Partido Socialista ganó 2.990 .935 votos, un $37.7 \%$ más que en los comicios de 2000. Estos nuevos votantes del PSOE, siguiendo las encuestas, tendrían distintas procedencias: a) antiguos votantes del PSOE que se habían mantenido en los últimos años en la abstención, y b) votantes que acudieron por primera vez a votar. Cabe recordar que, en estos comicios, había casi dos millones de jóvenes que habían cumplido los 18 años después del 2000.

- Mientras que el PP perdió votos respecto a las elecciones de 2000 en Andalucía, Cataluña, Galicia, Canarias, Madrid y Aragón, el PSOE aumentó su número de escaños en dichas comunidades autónomas. Asuntos como el Plan Hidrológico o El Prestige fueron temas a los que se les puede adjudicar parte del descenso de votos sufrido por el Partido Popular en Aragón y Galicia.

\section{Referencias bibliográficas}

- Agranoff, R; Meadow, R. G. (1989): "Political Campaigns", en Rice, R. E.; Atkin, C. K. (comp.) Public Communication Campaigns, California: Sage Publication, pp. 250-264.

- Alonso Hinojal, I. (1999): "La democracia mediatizada. Fuentes de conocimiento de los estados de opinión pública e información sobre encuestas”, en Ladevéze Núñez, L.; Sinova, J. (coords.): Política y medios de comunicación, Madrid: Fundación para el Análisis y los Estudios Sociales, pp. 1999-214.

- Artal, R.M. (2004): 11-M, 14-M: Onda expansiva, Madrid: Espejo de Tinta.

- Barreiro, B. (2004): “14-M: Elecciones a la sombra del terrorismo”, Claves de la Razón Práctica, n. ${ }^{141,}$ pp. 14-22.

- Berrocal, S. (2003): "La campaña electoral televisada: posibles influencias en el electorado", en Berrocal, S. (coord.): Comunicación política en televisión y nuevos medios, Barcelona: Ariel, pp. 135-162.

- Campmany, J. (2005): El efecto ZP, Barcelona: Planeta.

- Cacho, J. (1999): El negocio de la libertad, Madrid: Foca.

- Crespo, I. (dir.). (2002): Las campañas electorales y sus efectos en la decisión del voto. Vol. I, Valencia: Tirant lo Blanch.

- Crespo, I.; Moreno, C. (2003): “El estudio de las campañas electorales”, en Crespo, I. (dir.): Las campañas electorales y sus efectos en la decisión del voto. Vol. II, Valencia: Tirant lo Blanch, pp.19-44.

- Diccionario de la Lengua Española, (2001), Madrid: Real Academia Española.

- Díez Nicolás, J.; Semetko, H. A., (1999): "Los programas de noticias de televisión y las campañas electorales de 1993 y 1996: propiedad, contenido e influencia”, en Muñoz Alonso, A.; Rospir, J. I. (eds.): Democracia mediática y campañas electorales, Barcelona: Ariel Comunicación, pp. 151-201. 
- García-Abadillo, C. (2004): 11-M, La venganza, Madrid: La Esfera de los Libros.

- Martínez, A.; Méndez, M. (2004): “Los partidos: Agentes eficaces”, en Crespo, I. (dir.): Las campañas electorales y sus efectos en la decisión del voto. Vol.III, Valencia: Tirant lo Blanch, pp. 23-38.

- Worcester, R. (1995): “Los sondeos políticos y electorales”, en Muñoz Alonso, A.; Rospir, J.I. (eds.): Comunicación Política, Madrid: Universitas, pp.171-194.

\section{Hemerografía}

- $A B C$ (enero-marzo 2004).

- Barreiro, Belén: “En busca del votante perdido”, El País, 06/02/2004.

- Barreiro, Belén: “Habrá sorpresa el 14-M?, El País, 09/03/2004.

- Carbonel, Eudald: “La política en manos de los primates”, El Mundo, 23/02/2004.

• Ekaizer, Ernesto: “Doble factura: guerra y manipulación”, El País, 15/03/2004.

- El País (enero-marzo 2004).

- El Mundo, del siglo XXI (enero-marzo 2004).

- Marraco, Manuel: "El trasvase del Ebro diluye la ventaja de los <populares $>$ en Aragón”, El Mundo, 04/03/2004.

- Rodríguez, Jorge A.: "Dos millones de jóvenes elegirán por primera vez al jefe del Gobierno", El País, $20 / 02 / 2004$.

- Roig, Xavier: “El mensaje electoral”, El País, 17/02/2004.

- "El 66,1\% de los encuestados quiere que regresen las tropas de Irak", El País, 07/03/2004.

- "Los temas que el PP considera prioritarios acaparan las tertulias matinales de televisión", El País, 27/ $02 / 2004$.

\section{Encuestas}

CIS: Enero-Diciembre 2003; Enero-Diciembre 2004.

Gallup: Enero-Octubre 2004.

Instituto Opina: Enero-Septiembre 2004. 